\title{
Lipid profile an important risk factor in patients with ovarian tumors: a meta-analysis
}

\author{
Flaviu Ionut Faur ${ }^{1,2 *}$ Adelina $\mathrm{Clim}^{3}$, Ionel Nati ${ }^{3}$ Amadeus Dobrescu, ${ }^{1,2}$ \\ ${ }^{1}$ Surgery Clinic, Timisoara Emergency County Hospital, Romania \\ ${ }^{2}$ Victor Babes, University of Medicine and Pharmacy Timisoara Romania \\ ${ }^{3}$ Obstetric and Gynecology Clinic "Dominic Stanca” Cluj-Napoca, Romania \\ ${ }^{4}$ Iuliu Hatieganu, University of Medicine and Pharmacy Cluj-Napoca, Romania
}

*Correspondence: Flaviu Ionut Faur, Surgery Clinic, Timisoara Emergency County Hospital, Romania

Received date: November 25, 2021; Accepted date: December 10, 2020; Published date: January 07, 2022

Citation: Flaviu I. Faur, * Clim A, Nati I., Dobrescu A. (2022) Lipid profile an important risk factor in patients with ovarian tumors: a meta-analysis. J. Obstetrics Gynecology and Reproductive Sciences 6(2); DOI: 10.31579/2578-8965/108

Copyright: () 2022, Flaviu Ionut Faur, This is an open access article distributed under the Creative Commons Attribution License, which permits unrestricted use, distribution, and reproduction in any medium, provided the original work is properly cited.

\begin{abstract}
Introduction: In the literature ovarian tumors are knowed to be one of the most deadliest gynecological malignancies [1-4]. In US this type of malignancy represents $2.3 \%$ of all cancer-related death and about $4 \%$ of all new cancer cases among women. There are several studies that have reported the role of lipid profiles and it's role in ovarian tumorigenesis. Fatty acids are essential for cancer cells progression[4-8]. In our study we investigated the true differance in circulating lipid profiles (total cholesterol TC, triglyceride TG, high-density lipoprotein cholesterol HDL, low density lipoprotein cholesterol LDL) among patients with and without ovarian tumors (OT) using a meta-analytical approach.
\end{abstract}

Mehods: The meta-analysis was conducted using the MOOSE guidelines. PubMed, EMBASE and Cochrane Library were extensively searched ( with a period of publication restriction between 2007 and 2019) to indetify published studies using the following keywords: " total cholesterol ", "high-density lipoprotein", " triglycerides ", " low-density lipoprotein ", " ovarian cancer", " ovarian tumor", "lipid profile". The search methodology is shown in Fig. 1 and all references of retrieved articles were searched manually.

Results: Seven studies, involving 1542 OT cases and 2195 non-cases of OT were included in this meta-analysis and $\mathrm{I}^{2}$ statistics ranged between 97 and 99\%. Mean circulating TC and HDL were significantly lower among OT cases compared to non-OT cases $(P<0.04$ and $P<0.005)$.

Conclusion: There is a modest significant association between circulating HDL and risk of ovarian tumor but it is crucial to elucidate the implications of HDL in tumor manifestations and growth.

Keywords : ovarian tumor; lipid profile; total cholesterol; triglycerides; low-density lipoprotein

\section{Introduction}

In the literature ovarian tumors are knowed to be one of the most deadliest gynecological malignancies. In US this type of malignancy represents 2.3 $\%$ of all cancer-related death and about $4 \%$ of all new cancer cases among women. In the latest statistical cohort, there was an incidence of 11.6 cases/100.000 women per year, with an estimated 224.940 women living with this disease in the world. The simptomatology in ovarian tumors is hidden and about $70 \%$ of patients are diagnosed in advanced stage, wich underlines that ovarian tumors are a serious public health concern. As we all know lipids are biologically important hydrophobic moleculs vital for energy storage, cell signalling and maintenance of cell membrane integrity. Lipids are transported in the bloodstream with the aid of lipoprotein. Lipids are widely distributed in cellular organells and used as biologically vital active molecules. There are several studies that have reported the role of lipid profiles and it's role in ovarian tumorigenesis. Fatty acids are essential for cancer cells progression. In our study we investigated the true differance in circulating lipid profiles (total cholesterol TC, triglyceride TG, high-density lipoprotein cholesterol HDL, low density lipoprotein cholesterol LDL) among patients with and without ovarian tumors (OT) using a meta-analytical approach.

\section{Materials and methods}

The meta-analysis was conducted using the MOOSE guidelines. PubMed, EMBASE and Cochrane Library were extensively searched ( with a period 
of publication restriction between 2007 and 2019) to indetify published studies using the following keywords: " total cholesterol ", "high-density lipoprotein", " triglycerides ", " low-density lipoprotein ", " ovarian cancer", " ovarian tumor", "lipid profile". The search methodology is shown in Fig. 1 and all references of retrieved articles were searched manually.

\section{Study selection}

Criteria of inclusion in the meta-analysis:

- Case control studies in human population that investigated the association between lipid profile and ovarian tumors

- Studies that compared women with ovarian tumor with women without ovarian tumor

- $\quad$ Studies that compared lipid profile (TC, HDL, LDL, VLDL, TG) between women with ovarian tumor with women without ovarian tumor

The magnitude of variation across studies was assessed ussing $\mathrm{I}^{2}$ test statistics and a random-effects model was used to obtain mean estimates under considerable heterogeneity $\left(I^{2}\right.$-test $>50 \%$ or $P<0.05$ )

\section{Data extraction}

Name of authors, year of publication (after 2007), country, study population, sample size, lipid profile, criteria for case definition, mean values ( with standard deviation SD, standard error of mean SEM, confidence interval CI) of serum lipid profile (TC, LDL, HDL, TG) were extracted independently by two reviewers and differences in data extractions were resolved in recourse to a third reviewer. We establish that mean values of TC were transformed to $\mathrm{mg} / \mathrm{dl}$, but TG, HDL and LDL were transformed to mmol/L. Also, all values reported as SEM and CI were transformed into SD.

\section{Results}

We have done a literature research and indentified through electronic catalogue searching on PUBMED, EMBASE, COCHRANE library a number of 1704 studies from the primary ture search. About 384 were duplicates and we have excluded 1201 after examining titles and abstarcts. After full-text evaluation were excluded 112 studies and in this metaanalysis we included 7 studies (Table 1) that matched the inclusion criteria comprising 1542 ovarian tumor casess and 2195 non-ovarian tumor cases.

\begin{tabular}{|c|c|c|c|c|c|c|c|}
\hline Authors & Year & Country & Cases & Control & Lipid profile & $\begin{array}{l}\text { Ascertainment of ovarian } \\
\text { tumor cases }\end{array}$ & Classification \\
\hline Bukhari et al. & 2016 & Pakistan & 30 & 30 & $\begin{array}{l}\text { TC, TG, } \\
\text { HDL, LDL }\end{array}$ & Hospital/Medical record & NR \\
\hline Camuzcuoglu et al. & 2009 & Turkey & 24 & 29 & $\begin{array}{l}\text { TC, TG, } \\
\text { HDL, LDL }\end{array}$ & Hospital/Medical record & FIGO \\
\hline Delimaris et al. & 2007 & Greece & 15 & 30 & $\begin{array}{l}\text { TC, HDL, } \\
\text { LDL }\end{array}$ & Hospital confirmed & FIGO \\
\hline Chen et al. & 2017 & China & 573 & 1146 & TG, HDL & Histopathological examinations & FIGO \\
\hline Knapp et al. & 2017 & Poland & 74 & 81 & TC, TG & Hospital/Medical record & NR \\
\hline Melvin et al. & 2012 & Sweden & 786 & 829 & $\begin{array}{l}\text { TC, } \quad \text { TG, } \\
\text { HDL, LDL }\end{array}$ & Histopathological examinations & FIGO \\
\hline Qadir et al. & 2008 & Pakistan & 40 & 50 & $\begin{array}{l}\text { TC, } \quad \text { TG, } \\
\text { HDL, LDL }\end{array}$ & Hospital confirmed & FIGO \\
\hline
\end{tabular}

In our meta-analysis mean total cholesterol was significantly lower among ovarian tumors cases comapare to non-ovarian tumors cases $(P=0.04)$ also mean $\mathrm{HDl}=\mathrm{L}$ was significantly lower among OT cases compared to nonOT cases. All cases we stratified by age and this differences were insignificant. If we talk about mean TG and LDL yhe differences were insignificantly between OT and non-OT cases. As we mentioned we stratified our meta-analysis by age (Table 2). In table 2 we could observed that TG profile was significantly elevated among OT cases under 49 years old $0.059[0.54,0.63] \mathrm{mmol} / \mathrm{L} P<0.0001$ and contrariwise LDL profile was significantly elevated $0.38[0.23,0.06] \mathrm{mmol} / \mathrm{L} P<0.0001$ among OT cases over 49 years old.

In a subgroup of cases with malignant OT and/or advanced tumors TC was significantly lower $(P<0.05)$ and TC and LDL profiles were insignificantly different, but HDL profile was significanlty lower between OT and nonOT cases.

\begin{tabular}{|c|c|c|c|c|c|c|c|c|}
\hline & $\mathrm{TC}(\mathrm{mg} / \mathrm{dL})$ & D & TG (mmol/L) & D2 & HDL (mmol/L) & D3 & $\mathrm{LDL}(\mathrm{mmol} / \mathrm{L})$ & D4 \\
\hline All studies & $-16,52[-32,43 ;-0,72]$ & $\downarrow$ & $\left.\begin{array}{l}0.063 \\
0.28]\end{array}\right]-0.19$, & $\leftrightarrow$ & -0.24 & $\downarrow$ & $0.12[-0.23,-0.09]^{*}$ & $\leftrightarrow$ \\
\hline
\end{tabular}




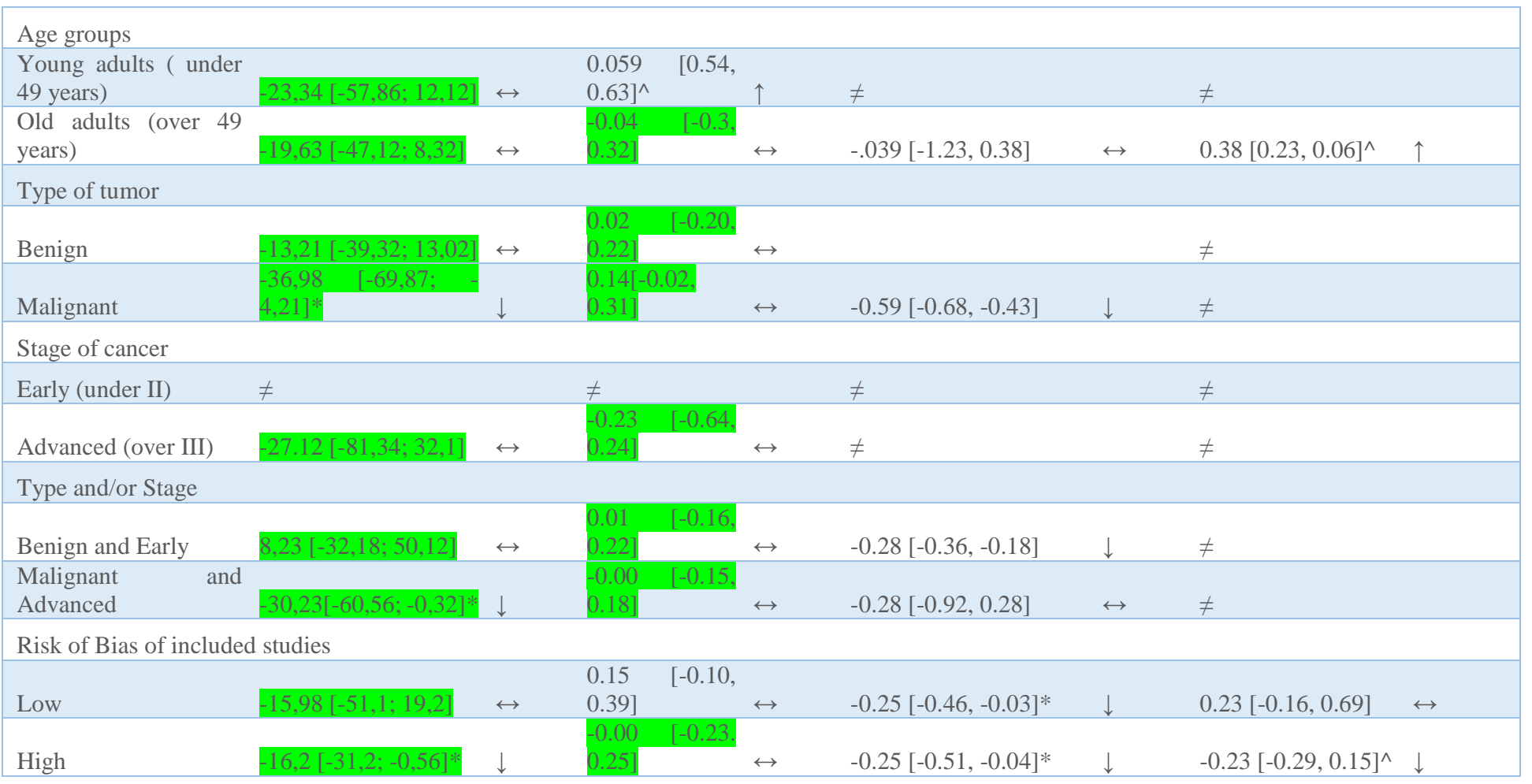

Mean difference and 95\% CI of Lipid Profile between cases and non-cases of ovarian tumors

D-direction of mean difference relative to non-ovarian tumour cases; TCTotal cholesterol; TG-Triglycerides; HDL-High density lipoprotein; LDLLow

density lipoprotein

$* \mathrm{p}<0.05$

$\wedge_{\mathrm{p}}<0.0001$

$\neq$ studies were insufficient to carry out the meta-analysis

$\uparrow$ mean difference significantly higher among cases than non-cases of ovarian tumour

$\downarrow$ mean difference significantly lower among cases than non-cases of ovarian tumour

$\leftrightarrow$ mean difference insignificantly different between cases than non-cases of ovarian tumour

\section{Discussion}

There are few metaanalytical studies in the literature about this topic. Lipid profil it is an important risk factor in ovarian tumorigenessis [9-14]. Total cholesterol and HDL profiles were significantly lower among patients with ovarian tumors but the profile level of TG and LDL were insignificant. Circulating lipid profiles are largely subject to alterations in occurrence of tumor events. In our meta-analysis we highlight the significance of lipids in OT outcomes. The strong affinity of cancer cells for sterols and lipids makes lipid metabolism a critical factor in cancer signaling and excessive production of lipogenic enzymes has been observed in several cancers and is linked with cancer severity and recurrance. In our meta-analysis we observed that HDL was inversely related to OT risk. This kind of observation was related by Gadomska et al. In a multidimensional analysis. As we all know the anti-inflammatory properties of HDL in inhibiting cell proliferatrion and apoptosis are crucial in preventing the oncreased intracellular oxidative stress that is a critical step in cancer pathogenesis $[15,16]$. Decreased HDl levels are associated with increased levels of proinflammatory cytokines including tumor necrosis factor-alpha and interleukin-6. Tumor cells express increased LDL receptor levels which lead to low LDL levels. Most studies included in our meta-analysis were cross-sectional and limited studies age-matched cases with controls in the eligible studies $[17,18]$.

\section{Conclusion}

There is a modest significant association between circulating HDL and risk of ovarian tumor but it is crucial to elucidate the implications of HDL in tumor manifestations and growth and it's important to highlights the role of serum lipid profile in diagnosis, prognosis and recurrence of the disease in future studies.

\section{Referances}

1. Anand P, Kunnumakkara AB, Sundaram C, Harikumar KB, Tharakan ST, Lai OS, et al. (2008) Cancer is a preventable disease that requires major lifestyle changes. Pharm Res Sep;25(9):20972116. 10.1007/s11095-008-9661-9

2. Aggarwal BB, Danda D, Gupta S, Gehlot P.(2009) Models for prevention and treatment of cancer: problems vs promises. Biochem Pharmacol Nov;78(9):1083-1094. 10.1016/j.bcp.2009.05.027

3. Lo AC, Soliman AS, El-Ghawalby N, Abdel-Wahab M, Fathy O, Khaled HM, et al.( 2007) Lifestyle, occupational, and reproductive factors in relation to pancreatic cancer risk. Pancreas . 10.1097/mpa.0b013e318053e7d3

4. Cvetkovic Z, Cvetkovic B, Petrovic M, Ranic M, DebeljakMartarcic J, Vucic V, et al. (2009). Lipid profile as a prognostic factor in cancer patients. J BUON Jul-Sep;14(3):501-506.

5. Muntoni S, Atzori L, Mereu R, Satta G, Macis MD, Congia M, et al. (2009) . Serum lipoproteins and cancer. Nutr Metab 
Cardiovasc

Dis

10.1016/j.numecd.2008.06.002

6. Singh S, Ramesh V, Premalatha B, Prashad KV, Ramadoss K. (2013). Alterations in serum lipid profile patterns in oral cancer. $J$ Nat Sci Biol Med . Jul;4(2):374-378. 10.4103/0976-9668.116994

7. Risch HA, Jain M, Marrett LD, Howe GR. (1994). Dietary fat intake and risk of epithelial ovarian cancer. J Natl Cancer Inst . Sep;86(18):1409-1415. 10.1093/jnci/86.18.1409

8. Llanos AA, Makambi KH, Tucker CA, Wallington SF, Shields PG, Adams-Campbell LL. (2012). Cholesterol, lipoproteins, and breast cancer risk in African American women. Ethn Dis ;22(3):281-287.

9. Kushi LH, Mink PJ, Folsom AR, Anderson KE, Zheng W, Lazovich D, et al.(1999) . Prospective study of diet and ovarian cancer. Am J Epidemiol . Jan;149(1):21-31. 10.1093/oxfordjournals.aje.a009723

10. La Vecchia C, Decarli A, Negri E, Parazzini F, Gentile A, Cecchetti G, et al. (1987). Dietary factors and the risk of epithelial ovarian cancer. J Natl Cancer Inst . Oct;79(4):663-669.

11. Knekt P, Steineck G, Järvinen R, Hakulinen T, Aromaa A. (1994) Intake of fried meat and risk of cancer: a follow-up study in Finland. Int $J \quad$ Cancer. Dec;59(6):756760.1002/ijc. 2910590608
12. Oliveras $\mathrm{G}$, et al. Novel anti-fatty acid synthase compounds with anti-cancer activity in HER2+ breast cancer. Ann N Y Acad Sci. 2010;1210:86-92.

13. Marz W, et al. HDL cholesterol: reappraisal of its clinical relevance. Clin Res Cardiol. 2017;106(9):663-75.

14. Morin EE, Li XA, Schwendeman A. (2018). HDL in Endocrine Carcinomas: Biomarker, Drug Carrier, and Potential Therapeutic. Front Endocrinol (Lausanne). 2018;9: 715.

15. Corbin IR, et al. (2013). Near-infrared fluorescent imaging of metastatic ovarian cancer using folate receptor-targeted highdensity lipoprotein nanocarriers. Nanomedicine (London, England).;8(6):875-90.

16. Shahzad MM, et al.(2011) Targeted delivery of small interfering RNA using reconstituted high-density lipoprotein nanoparticles. Neoplasia.;13(4): 309-19.

17. Murakami T, et al. (2010) Intracellular drug delivery by genetically engineered highdensity lipoprotein nanoparticles. Nanomedicine (Lond).;5(6):867-79.

18. Kim S, Keku TO, Martin C, Galanko J, Woosley JT, Schroeder JC, Satia JA, Halabi S, Sandler RS. (2008) Circulating levels of inflammatory cytokines and risk of colorectal adenomas. Cancer Res.68(1):323-8.

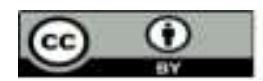

This work is licensed under Creative Commons Attribution 4.0 License

To Submit Your Article Click Here: Submit Manuscript

DOI: $10.31579 / 2578-8965 / 108$
Ready to submit your research? Choose Auctores and benefit from:

$>$ fast, convenient online submission

$>$ rigorous peer review by experienced research in your field

$>$ rapid publication on acceptance

$>$ authors retain copyrights

$>$ unique DOI for all articles

$>$ immediate, unrestricted online access

At Auctores, research is always in progress.

Learn more https://auctoresonline.org/journals/obstetrics-gynecology-andreproductive-sciences 